\title{
Impact of interfraction seroma collection on breast brachytherapy dosimetry - a mathematical model
}

\author{
Aashish Bhatt, MD', Keith Sowards, MS', Geetika Bhatt, MD', Andrew Freeman, MD², Anthony Dragun, MD' \\ University of Louisville School of Medicine, Lovisville, Kentucky, USA, 2Marshall University School of Medicine, Huntington, West Virginia, USA
}

\begin{abstract}
Purpose: Balloon brachytherapy is a widely accepted modality for delivery of accelerated partial breast irradiation (APBI). Our hypothesis was that inter-fraction seroma collection around the balloon surface would have an adverse effect on dosimetry of the target.

Material and methods: This is a dosimetric re-planning study using two volumetric models ( $30 \mathrm{cc}$ and $45 \mathrm{cc}$ ) in a Contura ${ }^{\circledR}$ multi-lumen balloon (MLB) catheter. In a previously treated patient, two customized baseline plans were generated using multiple channels of the Contura ${ }^{\circledR}$ catheter prescribed to the Planning Target Volume Evaluation (PTV_Eval). Symmetric expansions of $1.0 \mathrm{~mm}(0-9 \mathrm{~mm})$ increments around the balloon surface were performed to simulate a "Virtual Seroma" (VS) accumulation for both balloon volumes and plans were obtained for each expansion using Eclipse Brachyvision $^{\mathrm{TM}}$. An analysis of these plans was then performed to evaluate the effect of seroma accumulation on dosimetric parameters of V100 and V90.

Results: 20 plans were generated and analyzed (10 plans for each balloon volume), representing VS of 6.0-66.0 cc. There was a commensurate decrease in the dose delivered to the PTV_Eval V100 and V90 (as defined by the original treatment plan) with increasing VS accumulation leading to a sub-optimal coverage of the PTV_Eval. For 30 cc MLB catheter, V100 decreased by $1.4 \%$ and V90 decreased by $0.9 \%$ for every 1 cc of VS. For 45 cc MLB catheter, V100 decreased by $1.3 \%$ and V90 decreased by $1.15 \%$ for every 1.0 cc accumulation of VS.

Conclusions: Balloon catheter-tissue adherence ensures daily dose delivery to the planned PTV_Eval. Accumulation of seroma, hematoma or air between HDR fractions can significantly impact PTV_Eval dosimetry. Vacuum-port aspiration prior to delivery of each fraction, if available, should be considered to minimize the risk of geographic under dosing.
\end{abstract}

J Contemp Brachyther 2012; 4, 2: 101-105 DOI: $10.5114 /$ jcb.2012.29366

Key words: seroma, dosimetry, breast brachytherapy, contura catheter.

\section{Purpose}

Breast conservation surgery (BCS), followed by radiation therapy (RT) to the breast, is the preferred treatment for the majority of patients with early-stage breast cancer [1,2]. The overwhelming majority of local recurrences after BCS occur at or near the tumor bed [3-5] and pathologic studies suggest that mammographically detected cancers are rarely associated with microscopic extension of tumor away from the tumor bed $[6,7]$. These observations have led to the development and application of accelerated partial breast irradiation (APBI) in selected patients, where irradiation of the primary tumor bed, rather than the entire breast, may be sufficient to achieve durable local control after BCS.

The evidence for APBI using interstitial or balloon brachytherapy has been building with several phase II studies showing comparable in-breast tumor recurrence (IBTR) rates, as compared to EBRT techniques with better cosmetic outcomes [8-14]. Although diverse techniques to deliver APBI have been described [15], each approach requires accurate identification of target structures during the planning and prescription of therapy, as all subsequent dose delivery is based on these initial volumes.

Seroma, an abnormal collection of serous fluid in the dead space of the lumpectomy cavity following breast-conserving surgery, is the most common post surgical complication with reported rates of $15-76 \%$ [16-18]. The pathophysiology and risk factors for seroma formation are not fully understood, but it generally occurs in the acute postoperative period. Seroma, hematoma and/or air may therefore collect in the balloon-tissue interface following placement of a balloon brachytherapy catheter and during a course of fractionated high-dose-rate (HDR) therapy. If not addressed, inter-fraction seroma development may ultimately interfere with dose delivery to the lumpectomy target tissue, unless the patient is imaged prior to each treatment and the dosimetry corrected. In this study, we endeavor to quantify the possible impact of seroma accumulation using dosimetric modeling in patients treated with Contura ${ }^{\circledR}$ (SenoRx, Inc., Irvine, CA) multilumen balloon (MLB) brachytherapy. 


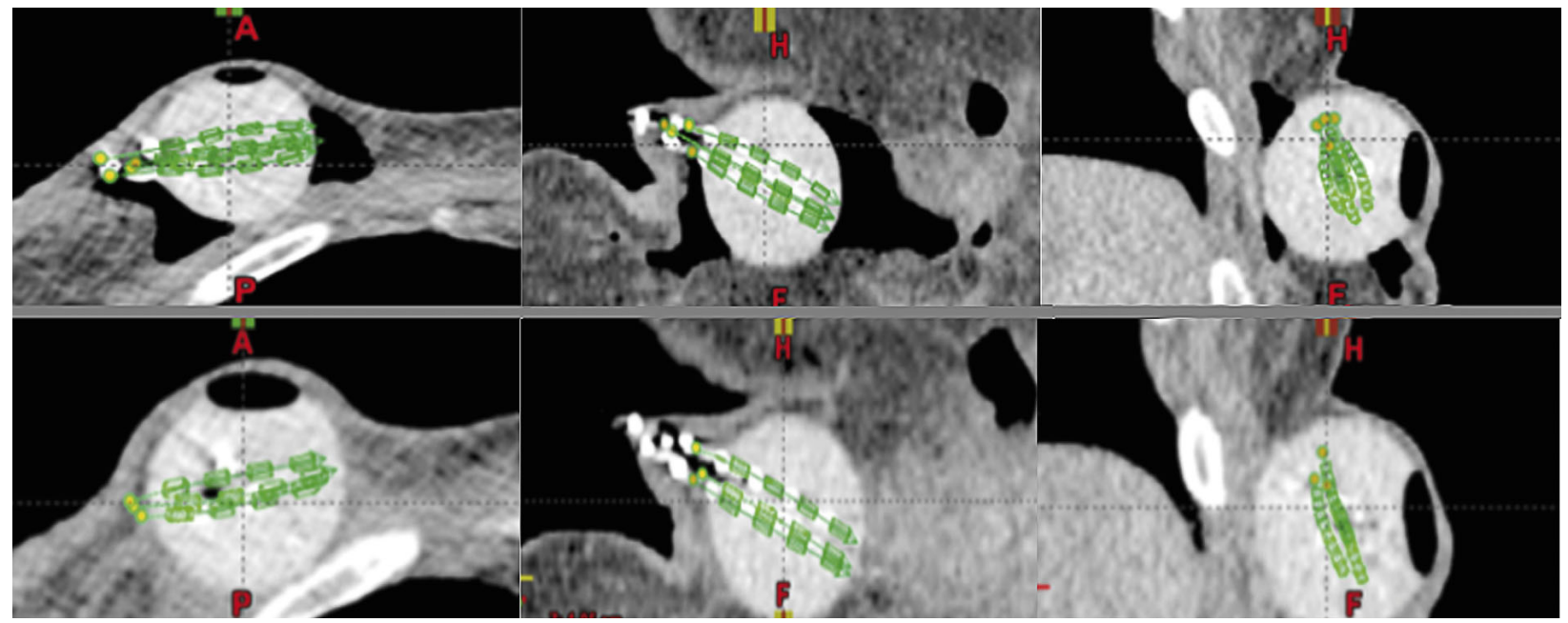

Fig. 1. Pre (upper row) and post (lower row) seroma aspiration images at time of CT-simulation. Note improved balloon to tissue adherence

\section{Material and methods}

This is a dosimetric re-planning study using two volumes (30 cc and $45 \mathrm{cc}$ ) in a Contura ${ }^{\circledR}$ MLB catheter model. The Contura ${ }^{\circledR}$ balloon catheter was used for this study, because it is the only FDA-approved balloon brachytherapy device currently on the market that contains a vacuum port specifically designed to aspirate seroma, hematoma and/or air collection. Proof of principle of aspiration of the accumulated seroma via the vacuum port is shown in Fig. 1.

Two customized optimal treatment plans (for balloon volumes of 30 and $45 \mathrm{cc}$ ) were first generated using a single patient scan. Uniform balloon expansion of $1 \mathrm{~mm}$ increments around the balloon surface was then performed to simulate a theoretical and symmetrical "virtual seroma" (VS) accumulation up to $9 \mathrm{~mm}$ (Fig. 2). The volume (in cc) of this expansion was calculated by the Eclipse Brachyvision ${ }^{\mathrm{TM}}$ (Varian Inc., Palo Alto, CA) software to estimate each VS. With the use of the multiple (up to 5) channels of the Contura ${ }^{\circledR}$ catheter, the plans were generated prescribing 3.4 Gy per fraction to a "PTV_Eval" (a $10 \mathrm{~mm}$ PTV with appropriate modifications for skin and chest wall per RTOG 0413 guidelines).

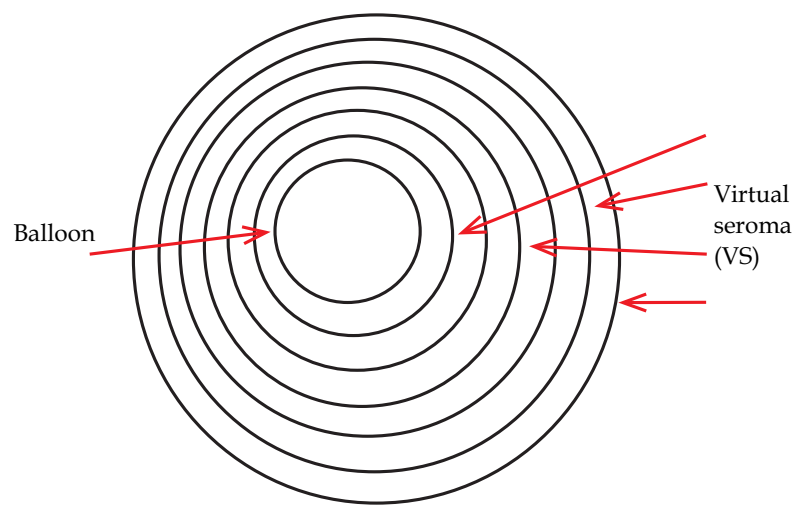

Fig. 2. Model of Virtual seroma (VS) accumulation used for calculations
New individualized plans were then generated for each $1 \mathrm{~mm}$ increment, representing additional accumulating VS, resulting in 10 plans for each balloon volume. Each potential plan scenario and dose-volume histograms were individually generated to compare changes in dosimetric coverage of the initially defined PTV_Eval. PTV_Eval dosimetry was then analyzed independently for each plan to assess the effect of the VS on the percent volume of PTV_Eval covered by the 100\% isodose line (IDL) (V100) and the percent volume of PTV_Eval covered by the $90 \%$ IDL (V90).

\section{Results}

A total of twenty individual and customized 3-dimensional brachytherapy plans were generated for this comparative dosimetric study (ten each for $30 \mathrm{cc}$ and $45 \mathrm{cc}$ balloon volumes), representing VS volumes of 6-60 cc. For each simulated plan, the accumulation of VS volume resulted in increased distance separating peri-cavity breast tissue target from the balloon surface. This separation produced a commensurate decrease in the V100 and the V90 of the PTV_Eval (as defined by the original treatment plan) with increasing VS accumulation. Table 1 shows the effect of increasing seroma accumulation on the plans generated for $30 \mathrm{cc}$ and $45 \mathrm{cc}$ balloon, respectively. Each increasing uniform expansion of the balloon catheter to simulate VS resulted in a symmetrical volumetric target separation. This translated into a corresponding decrease in V100 and V90. Tabular data is represented as a scatter plot for $30 \mathrm{cc}$ and $45 \mathrm{cc}$ balloon catheters in Figs. 3, 4 and 5, 6, respectively. The best-fit-line model for $30 \mathrm{cc}$ balloon showed that V100 coverage of the PTV_EVAL decreased by $1.4 \%$ for every $1 \mathrm{cc}$ accumulation of VS (Range $=7-10 \%$ for every $5-7 \mathrm{cc}$ ). A similar pattern was noted for the scatter plot analysis for 45 cc catheter, where V100 was shown to decrease by $1.3 \%$ for every $1 \mathrm{cc}$ accumulation of VS (Range $=6-9 \%$ for every 5-7 cc). For V90, scatter plot analyses revealed a decreased coverage of $0.9 \%$ for every $1 \mathrm{cc}$ of VS for $30 \mathrm{cc}$ balloon plans and $1.15 \%$ for 45 cc balloon plans. 
Table 1. Twenty plans based on 30 and $45 \mathrm{cc}$ balloon catheters

\begin{tabular}{|c|c|c|c|c|c|c|c|c|c|}
\hline \multirow[t]{2}{*}{ Plan \# } & \multirow[b]{2}{*}{$\begin{array}{l}\text { Increase } \\
\text { in balloon } \\
\text { to breast } \\
\text { tissue } \\
\text { distance } \\
(\mathrm{mm})\end{array}$} & \multicolumn{4}{|c|}{$30 \mathrm{cc}$ balloon catheter plans } & \multicolumn{4}{|c|}{$45 \mathrm{cc}$ balloon catheter plans } \\
\hline & & $\begin{array}{l}\text { Seroma } \\
\text { volume } \\
\text { (cc) }\end{array}$ & $\begin{array}{c}\text { Vol of } \\
\text { PTV_Eval } \\
\text { covered } \\
\text { by } 100 \% \\
\text { IDL (cc) }\end{array}$ & $\begin{array}{c}\% \text { of initial } \\
\text { PTV_Eval } \\
\text { covered } \\
\text { by } 100 \% \\
\text { IDL (V100) }\end{array}$ & $\begin{array}{c}\text { \%PTV_Eval } \\
\text { covered } \\
\text { by } 90 \% \\
\text { IDL (V90) }\end{array}$ & $\begin{array}{l}\text { Seroma } \\
\text { volume } \\
\text { (cc) }\end{array}$ & $\begin{array}{c}\text { Vol of } \\
\text { PTV_Eval } \\
\text { covered } \\
\text { by } 100 \% \\
\text { IDL (cc) }\end{array}$ & $\begin{array}{c}\% \text { of initial } \\
\text { PTV_Eval } \\
\text { covered } \\
\text { by } 100 \% \\
\text { IDL (V100) }\end{array}$ & $\begin{array}{c}\text { \%PTV_Eval } \\
\text { covered } \\
\text { by } 90 \% \\
\text { IDL (V90) }\end{array}$ \\
\hline 1 & 0 & 0 & 65.6 & 100 & 100 & 0 & 73.5 & 100 & 99.8 \\
\hline 2 & 1 & 6 & 59.6 & 91 & 99.6 & 6.8 & 66.7 & 91 & 97.9 \\
\hline 3 & 2 & 10.3 & 55.3 & 84 & 99.2 & 11.6 & 61.9 & 84 & 93.3 \\
\hline 4 & 3 & 15.7 & 49.9 & 76 & 97.3 & 17.9 & 55.6 & 76 & 84.7 \\
\hline 5 & 4 & 21.8 & 43.8 & 67 & 91.4 & 25 & 48.5 & 66 & 73.5 \\
\hline 6 & 5 & 28.2 & 37.4 & 57 & 81.8 & 32.2 & 41.3 & 56 & 62.9 \\
\hline 7 & 6 & 34.9 & 30.7 & 47 & 71.2 & 40 & 33.5 & 46 & 52.3 \\
\hline 8 & 7 & 41.8 & 23.8 & 36 & 61.0 & 47.8 & 25.7 & 35 & 42.7 \\
\hline 9 & 8 & 49.3 & 16.3 & 25 & 50.8 & 56.1 & 17.4 & 24 & 33.2 \\
\hline 10 & 9 & 59.2 & 9.9 & 15 & 40.5 & 66.6 & 6.9 & 9 & 23.7 \\
\hline
\end{tabular}

Note the effect of accumulating seroma causing an increase in balloon to breast tissue distance. This in turn affects the volume and percentage of the initial PTV_Eval covered by 100\% (V100) and 90\% (V90) IDL leading to sub-optimal plans. IDL - isodose line

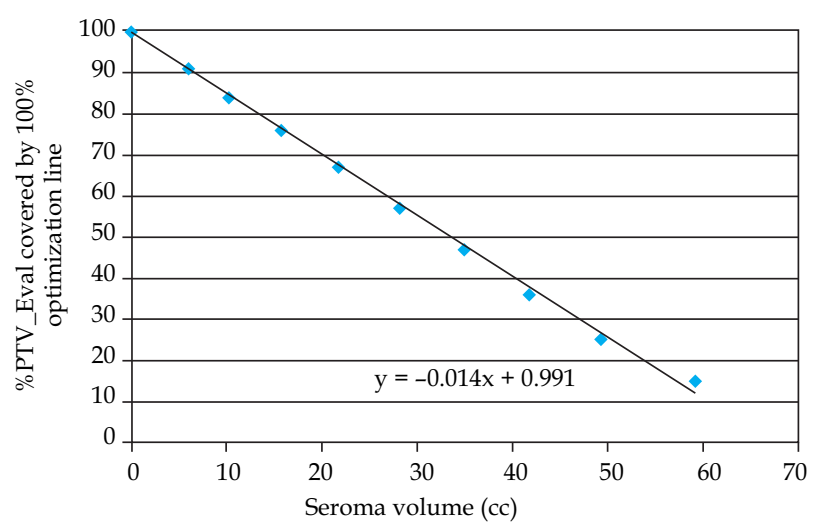

Fig. 3. Scatter plot and best fit line of V100 for repeat plans with increasing seroma volumes for $30 \mathrm{cc}$ balloon catheter

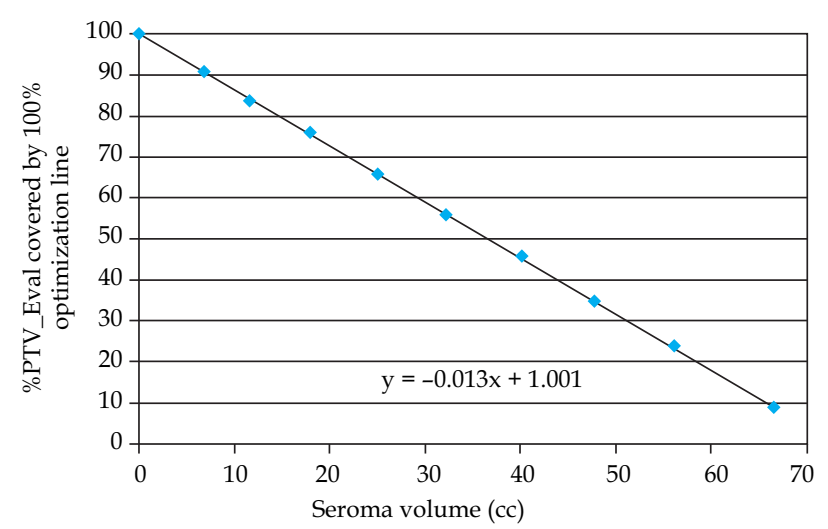

Fig. 5. Scatter plot and best fit line of V100 for repeat plans with increasing seroma volumes for $45 \mathrm{cc}$ balloon catheter

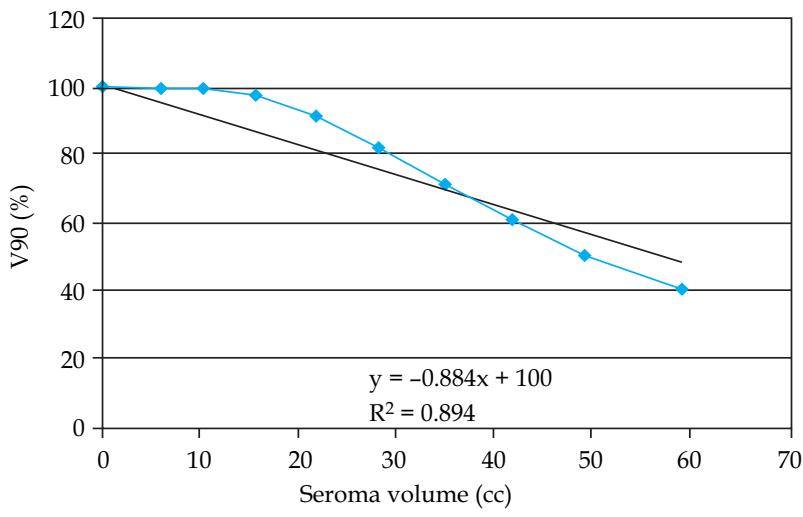

Fig. 4. Scatter plot and best fit line of V90 for repeat plans with increasing seroma volumes for $30 \mathrm{cc}$ balloon catheter

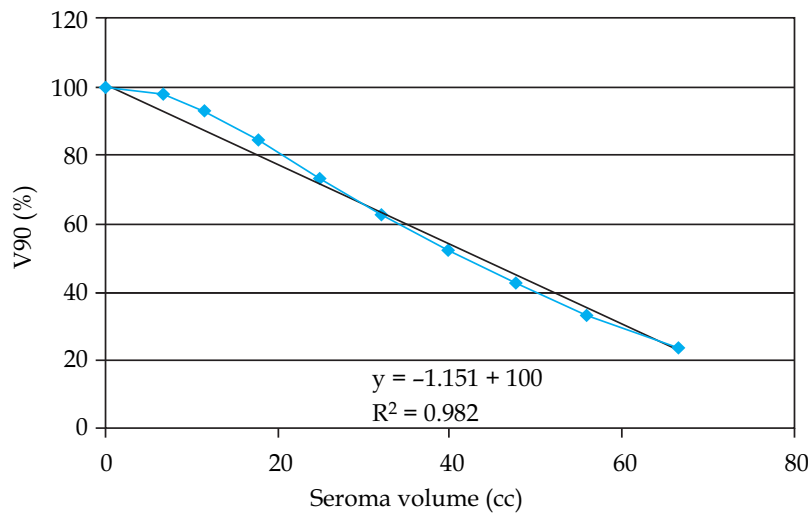

Fig. 6. Scatter plot and best fit line of V90 for repeat plans with increasing seroma volumes for $45 \mathrm{cc}$ balloon catheter 


\section{Discussion}

Seroma is formed by acute inflammatory exudates in response to surgical trauma and acute phase of wound healing. Although the pathophysiology of seroma is incompletely understood, the formation seems to be correlated with certain cytokine levels and body mass index [11,19]. With regards to breast brachytherapy, post-treatment seroma formation has been shown to be more likely to result from "open" (intra-operative) versus "closed" (post-operative) placement of the balloon brachytherapy catheter. Soran et al. [20] showed a $20 \%$ rate of persistent seroma (> 6 months) with open placement versus $0 \%$ for post-operative placement. Cosmetic outcomes were also noted to be worse in patients with seroma. Watkins et al. [21] showed that the only factor identified as statistically significant for the development of any seroma was catheter placement on a day of resection vs. $\geq 1$ day later ( $59 \%$ vs. $33 \%$ ). Numerous recent imaging studies have shown that the lumpectomy cavity volume changes significantly in time frames relevant to radiotherapy planning and delivery [16,17,22-27]. The range of average volume changes reported in these studies was $22.5-64 \%[16,27]$. The change in volume of the seroma has been shown to affect the RT boost volumes with EBRT [22,25]. Huh et al. [24] investigated the inter-fractional dose variation that occurs during EBRT when a seroma is drained during treatment. They found that the resultant change in anatomy after seroma drainage led to significant effects on dose homogeneity in the treatment volume, up to $13.9 \%$ for conventional treatment and $20.7 \%$ for IMRT treatment. Data suggests that the immediate postoperative course was the time during which the greatest and most rapid change occurs within the tumor bed [28]. The volume of seroma formation is highest in the first 8 weeks following surgery, the usual time frame when breast brachytherapy is delivered. A study by Kader et al. [26] showed that the mean seroma volume decreased from $47 \mathrm{~cm}^{3}$ in weeks 3-4 to $30 \mathrm{~cm}^{3}$ during weeks 7-8 after surgery.

The purpose of our study was to quantify the potential effect of inter-fraction seroma collection around the balloon surface on the dosimetry of the lumpectomy cavity target volume (PTV_Eval). Our analysis shows a concerning trend for sub-optimal coverage of target tissue with inter-fraction seroma accumulation, which may be under appreciated in routine clinical practice. With the potential for under dosing of tissue at highest risk for recurrence, seroma accumulation is a critical factor that warrants consideration throughout the entire process of brachytherapy treatment planning and delivery. One practical limitation of our study is that in a clinical setting, the seroma collection tends to be asymmetrical around the balloon catheter. This study results may not thus directly apply to current clinical practice, but adds credence to the hypothesis regardless, since the concept utilized is a spherical volume model. The effect on a dose volume histogram would not change if the absolute volume was symmetric or asymmetric.

Increased seroma formation would most likely lead to increased lubrication of the cavity and separation of the balloon from cavity walls, as well as the rotation of the balloon in the cavity. Vice versa, increased rotational motion could also be an indication of seroma collection. Even if aspirat- ed and the device is fixed in the cavity, it is important to verify that it has not rotated from the planned position, especially if there is an asymmetric loading. This can be easily achieved by marking the balloon catheter and the skin adjacent to it and checking the alignment of the two marks prior to each treatment. Aspiration before therapy could eliminate the need for pre-treatment repeat CT-imaging and re-planning in the sub-group of patients with excessive seroma accumulation. This would eliminate excess unwanted radiation to the patient and also reduce CT time. However, an ultrasound must be performed to verify balloon integrity prior to each fraction.

\section{Conclusions}

Accumulation of seroma, hematoma or air between HDR fractions can have a significant negative impact on PTV_Eval dosimetry (V100 \& V90) and worsen balloon catheter-tissue interface. Our study suggests that inter-fraction aspiration of accumulated seroma during a course of fractionated high-doserate balloon brachytherapy may decrease variability in target dosimetry while also offering better fidelity to the initial CT-based treatment plan. Aspiration prior to each fraction could help minimize seroma interference with target dosimetry. However, not all seroma can be expected to be removed by vacuum-port aspiration. In such instances, dose-shaping with a multi-lumen catheter has the potential to provide superior coverage of the target breast tissue.

\section{Information}

Parts of this work were presented as a "Poster Discussion" at the annual meeting of the American Brachytherapy Society (ABS) held in Atlanta, USA.

\section{References}

1. Whelan T, Olivotto I, Levine M. Clinical practice guidelines for the care and treatment of breast cancer: breast radiotherapy after breast-conserving surgery (summary of the 2003 update). CMAJ 2003; 168: 437-439.

2. Adjuvant therapy for breast cancer. NIH Consens Statement 2000; 17: 1-35.

3. Veronesi U, Marubini E, Mariani L et al. Radiotherapy after breast-conserving surgery in small breast carcinoma: long-term results of a randomized trial. Ann Oncol 2001; 12: 997-1003.

4. Clark RM, McCulloch PB, Levine MN et al. Randomized clinical trial to assess the effectiveness of breast irradiation following lumpectomy and axillary dissection for node-negative breast cancer. I Natl Cancer Inst 1992; 84: 683-689.

5. Liljegren G, Holmberg L, Bergh J et al. 10-year results after sector resection with or without postoperative radiotherapy for stage I breast cancer: a randomized trial. J Clin Oncol 1999; 17: 2326-2333.

6. Imamura H, Haga S, Shimizu T et al. Relationship between the morphological and biological characteristics of intraductal components accompanying invasive ductal breast carcinoma and patient age. Breast Cancer Res Treat 2000; 62: 177-184.

7. Faverly DR, Burgers L, Bult P et al. Three dimensional imaging of mammary ductal carcinoma in situ: clinical implications. Semin Diagn Pathol 1994; 11: 193-198.

8. King TA, Bolton JS, Kuske RR et al. Long-term results of widefield brachytherapy as the sole method of radiation therapy after segmental mastectomy for $\mathrm{T}(\mathrm{is}, 1,2)$ breast cancer. Am J Surg 2000; 180: 299-304. 
9. Polgar C, Sulyok Z, Fodor J et al. Sole brachytherapy of the tumor bed after conservative surgery for T1 breast cancer: five-year results of a phase I-II study and initial findings of a randomized phase III trial. J Surg Oncol 2002; 80: 121-128; discussion 129.

10. Perera F, Engel J, Holliday R et al. Local resection and brachytherapy confined to the lumpectomy site for early breast cancer: a pilot study. J Surg Oncol 1997; 65: 263-267; discussion 267-268.

11. Perera F, Chisela F, Engel J et al. Method of localization and implantation of the lumpectomy site for high dose rate brachytherapy after conservative surgery for T1 and T2 breast cancer. Int J Radiat Oncol Biol Phys 1995; 31: 959-965.

12. Vicini FA, Baglan KL, Kestin LL et al. Accelerated treatment of breast cancer. J Clin Oncol 2001; 19: 1993-2001.

13. Baglan KL, Martinez AA, Frazier RC et al. The use of highdose-rate brachytherapy alone after lumpectomy in patients with early-stage breast cancer treated with breast-conserving therapy. Int J Radiat Oncol Biol Phys 2001; 50: 1003-1011.

14. Krishnan L, Jewell WR, Tawfik OW et al. Breast conservation therapy with tumor bed irradiation alone in a selected group of patients with stage I breast cancer. Breast J 2001; 7: 91-96.

15. Arthur DW, Vicini FA. Accelerated partial breast irradiation as a part of breast conservation therapy. J Clin Oncol 2005; 23: 1726-1735.

16. Gonzalez EA, Saltzstein EC, Riedner CS et al. Seroma formation following breast cancer surgery. Breast J 2003; 9: 385-388.

17. Woodworth PA, McBoyle MF, Helmer SD et al. Seroma formation after breast cancer surgery: incidence and predicting factors. Am Surg 2000; 66: 444-450; discussion 450-441.

18. Evans SB, Kaufman SA, Price LL et al. Persistent seroma after intraoperative placement of MammoSite for accelerated partial breast irradiation: incidence, pathologic anatomy, and contributing factors. Int J Radiat Oncol Biol Phys 2006; 65: 333-339.

19. Chow LW, Loo WT, Yuen KY et al. The study of cytokine dynamics at the operation site after mastectomy. Wound Repair Regen 2003; 11: 326-330.

20. Soran A, Evrensel T, Beriwal S et al. Placement technique and the early complications of balloon breast brachytherapy: Magee-Womens Hospital experience. Am J Clin Oncol 2007; 30: 152-155.

21. Watkins JM, Harper JL, Dragun AE et al. Incidence and prognostic factors for seroma development after MammoSite breast brachytherapy. Brachytherapy 2008; 7: 305-309.

22. Sharma R, Spierer M, Mutyala $S$ et al. Change in seroma volume during whole-breast radiation therapy. Int J Radiat Oncol Biol Phys 2009; 75: 89-93.

23. Oh KS, Kong FM, Griffith KA et al. Planning the breast tumor bed boost: changes in the excision cavity volume and surgical scar location after breast-conserving surgery and whole-breast irradiation. Int J Radiat Oncol Biol Phys 2006; 66: 680-686.

24. Huh SJ, Han Y, Park W et al. Interfractional dose variation due to seromas in radiotherapy of breast cancer. Med Dosim 2005; 30: 8-11.

25. Tersteeg RJ, Roesink JM, Albregts $M$ et al. Changes in excision cavity volume: prediction of the reduction in absolute volume during breast irradiation. Int J Radiat Oncol Biol Phys 2009; 74: 1181-1185.

26. Kader HA, Truong PT, Pai R et al. When is CT-based postoperative seroma most useful to plan partial breast radiotherapy? Evaluation of clinical factors affecting seroma volume and clarity. Int J Radiat Oncol Biol Phys 2008; 72: 1064-1069.

27. Wong EK, Truong PT, Kader HA et al. Consistency in seroma contouring for partial breast radiotherapy: impact of guidelines. Int J Radiat Oncol Biol Phys 2006; 66: 372-376.
28. Prendergast B, Indelicato DJ, Grobmyer SR et al. The dynamic tumor bed: volumetric changes in the lumpectomy cavity during breast-conserving therapy. Int J Radiat Oncol Biol Phys 2009; 74: 695-701. 